\title{
OMAE2013-10240
}

\section{TWO-DIMENSIONAL COUPLED VORTEX-INDUCED VIBRATION OF CIRCULAR CYLINDER: PREDICTION AND EXTRACTION OF HYDRODYNAMICS PROPERTIES}

\author{
Hossein Zanganeh, Narakorn Srinil ${ }^{\star}$ \\ Department of Naval Architecture and Marine Engineering, University of Strathclyde \\ Glasgow, Scotland, UK, "corresponding author: narakorn.srinil@strath.ac.uk
}

\begin{abstract}
An advanced model for predicting a two-dimensional coupled cross-flow and in-line vortex-induced vibration (VIV) of a flexibly-mounted circular cylinder in a uniform flow is proposed and investigated. Attention is placed on a systematic extraction of variable hydrodynamics properties associated with a bi-directional fluid-structure interaction system. The governing equations of motion are based on double Duffing-van der Pol (structural-wake) oscillators with the two structural equations containing cubic and quadratic nonlinear terms. The cubic nonlinearities capture the geometrical coupling of crossflow/in-line displacements excited by hydrodynamic lift/drag forces whereas the quadratic nonlinearities allow fluid-structure interactions. The combined analytical and numerical solutions of the proposed model are established. By varying flow velocities in numerical simulations, the derived low-order model qualitatively captures several key VIV characteristics of coupled in-line/cross-flow oscillations. By making use of a newly-derived empirical formula, the predicted maximum crossflow/in-line VIV amplitudes and associated lock-in ranges compare well with several experimental results for cylinders with low/high mass or damping ratios. Moreover, such important hydrodynamic properties as VIV-induced mean drag, added mass, excitation and damping terms can be systematically determined via the proposed model and compared well with some experimental results in the literature.
\end{abstract}

\section{INTRODUCTION}

Vortex-induced vibration (VIV) is a basic phenomenon commonly encountered in various practical engineering applications and physical sciences where a fluid flow dynamically excites and interacts with a bluff solid/flexible structure. In connection with the oil \& gas industry, the risk of VIV is one of the most technically and economically critical concerns in the analysis and design of key offshore cylindrical structures including risers, mooring cables, pipelines and subsea components. Although a basic mechanism of the VIV occurrence is well known (Blevins, 1990) and VIV-related subjects have been extensively investigated (Bearman, 2011; Sarpkaya, 2004; Williamson and Govardhan, 2004), a completely reliable simulation model for predicting the associated fluid-structure interaction and nonlinear dynamical behavior is still needed. Owing to the complexity of the vortex hydrodynamics, the intrinsic mechanism of the structure, the overall elasto-hydro nonlinearities, the influence of several mechanical/physical parameters, and the necessity to calibrate and validate the simulation model with substantial experimental data, modelling of VIV remains a challenging theme.

VIV occurrences are widely categorized as cross-flow or in-line VIV in which the structure oscillates in the direction transverse to or aligned with the flow, respectively. Many studies have focused on the modelling of pure cross-flow VIV excited by the lift force because of its usually observed largest response (Gabbai and Benaroya, 2005; Sarpkaya, 2004). Very little is known about the effect of oscillating drag force, the ensuing in-line VIV, the coupling of cross-flow/in-line VIV, the dependence on system parameters and how to realistically model these features. Several recent experimental studies have evidenced the significant effect of in-line VIV (Dahl et al., 2006; Dahl et al., 2010; Jauvtis and Williamson, 2004); due to a doubled oscillating frequency, this can contribute - as much as the cross-flow VIV - to the current-induced fatigue damage of structures (Vandiver and Jong, 1987). Computational flow visualizations have also illustrated different vortex mode patterns in the wake behind cylinders oscillating with onedegree-of-freedom (DOF) vs. two-DOF displacements. Due to combined lift/drag forces associated with the shedding vortices and the fact that actual underwater structures possess multiple natural frequencies in different directions, a condition of coupled cross-flow/in-line VIV is certainly achievable in most 
practical situations which can be responsible for dangerouslyamplified dynamics. Nevertheless, most of the numerical tools currently used in the engineering industry are limited to the analysis of cross-flow-only VIV (Chaplin et al., 2005; Srinil, 2010, 2011; Srinil et al., 2009). Therefore, an advanced predictive model accounting for the coupled cross-flow/in-line, two-dimensional (2-D) or 2-DOF VIV would be worthwhile from a practical and industrial viewpoint.

Insights into the hydrodynamic properties (such as added mass, added damping and mean drag) of cylinder 2-DOF VIV are also important from an analysis and design viewpoint since these properties could be further applied to the analysis of actual flexible cylinders including marine risers, cables and mooring lines. However, a systematic approach in conjunction with an advanced mathematical modelling to extract these properties is still lacking in the literature.

This study presents an advanced model and combined numerical and analytical approach to extract hydrodynamic properties from the 2-DOF VIV of circular cylinders, by also capturing the important effect of system mass, damping and natural frequency ratios. Some initial comparisons with known experimental results are made and discussed along with several parametric studies in the case of varying flow velocities.

\section{COUPLED CROSS-FLOW/IN-LINE VIV MODEL}

The capability to reasonably model and accurately predict the coupled cross-flow/in-line VIV structural response excited by the unsteady flow field has been a major challenge to modelers and offshore engineers for many years although some prediction tools are currently available in the industry.

A schematic model of the cylinder subject to a uniform flow $V$ and restrained by two pairs of springs to oscillate in $\mathrm{X}$ and $\mathrm{Y}$ directions is displayed in Fig. 1a. The key aspect in the formulation of system equations of motions is to capture the quadratic relationship between in-line and cross-flow displacements (Vandiver and Jong, 1987). Following Wang et al. (2003), the two-directional unsteady fluid forces can be exerted on the oscillating cylinder as opposed to the stationary one, by also accounting for the relative velocities between the incoming flow and the cylinder in-line motion. As a result, the sectional lift $\left(F_{L}\right)$ and drag $\left(F_{D}\right)$ forces coincide with an arbitrary plane making up an angle of $\theta$ with respect to the $\mathrm{Y}$ and $\mathrm{X}$ axes, respectively.

Two cases can be realized depending on whether $\theta$ is counterclockwise (Fig. 1b) or clockwise (Fig. 1d). From our numerical simulation experience, it has been discovered that such $\theta$ direction plays a key role in the ensuing phase difference between cross-flow and in-line oscillations and, in turn, the figure-of-eight appearing shape. In general, the orbital plot exhibits a figure-eight trajectory with tips pointing upstream with a counterclockwise $\theta$ model (e.g. Fig. 1c) or downstream with a clockwise $\theta$ model (e.g. Fig. 1e). As both cases have been experimentally observed in the literature, they are herein accounted for in the generalized model formulation.

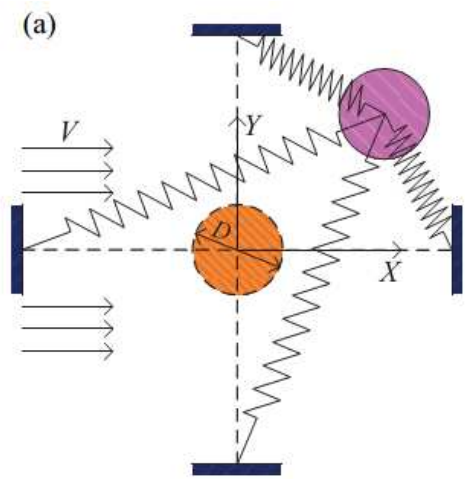

(b)
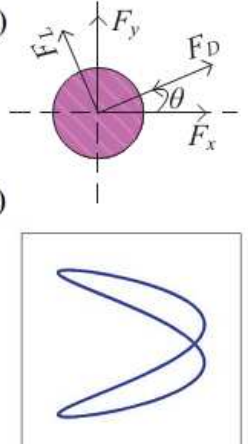

(d)

(e)
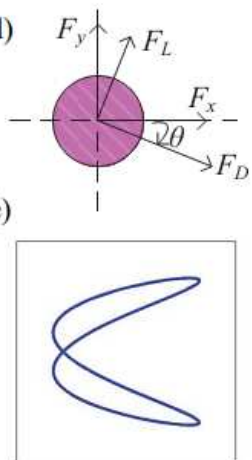

Figure 1 Model for a 2-DOF VIV of circular cylinder

By assuming a small $\theta$, the unsteady hydrodynamic forces $F_{x}$ and $F_{y}$ may be simplified after resolving $F_{L}$ and $F_{D}$ into the $\mathrm{X}$ and $\mathrm{Y}$ directions as

$$
\begin{aligned}
& F_{x}=F_{D} \cos \theta \mp F_{L} \sin \theta \approx F_{D} \mp F_{L} \dot{\tilde{Y}} / V, \\
& F_{y}=F_{L} \cos \theta \pm F_{D} \sin \theta \approx F_{L} \pm F_{D} \dot{\tilde{Y}} / V,
\end{aligned}
$$

where $\dot{\tilde{Y}}$ is the dimensional transverse displacement, a dot denotes differentiation with respect to the dimensional time $T$, $\rho$ is the fluid density, $C_{D}$ and $C_{L}$ are the time-varying drag and lift coefficients, the minus (positive) and positive (minus) sign in Eq. (1) (Eq. 2) correspond to the case of counterclockwise and clockwise $\theta$, respectively.

By assigning the fluid vortex variables as $p=2 C_{D} / C_{D 0}$ and $q=2 C_{L} / C_{L O}$ (Facchinetti et al., 2004) in which $C_{D O}$ and $C_{L O}$ are the associated drag and lift coefficients of a stationary cylinder (assumed as $C_{D O}=0.2$ (Currie and Turnbull, 1987) and $C_{L O}=0.3$ (Blevins, 1990)), the time variation of $p$ and $q$ may be assumed to follow the self-excitation and -limiting mechanism of the van der Pol wake oscillators. By introducing the dimensionless time $t=\omega_{n y} T$ and normalizing the displacements with respect to $D$, the nonlinearly coupled equations describing the in-line $(x)$ and cross-flow $(y)$ oscillations of the cylinder subject to the fluctuating fluid force components $(p, q)$ may be expressed in dimensionless form as (Srinil and Zanganeh, 2012)

$$
\begin{aligned}
& \ddot{x}+\lambda_{x} \dot{x}+f^{2}\left(x+\alpha_{x} x^{3}+\beta_{x} x y^{2}\right)=M_{D} \Omega^{2} p \mp 2 \pi M_{L} \Omega^{2}\left(q \dot{y} / V_{r}\right), \\
& \ddot{p}+2 \varepsilon_{x} \Omega\left(p^{2}-1\right) \dot{p}+4 \Omega^{2} p=\Lambda_{x} \ddot{x},
\end{aligned}
$$


$\ddot{y}+\lambda_{y} \dot{y}+y+\alpha_{y} y^{3}+\beta_{y} y x^{2}=M_{L} \Omega^{2} q \pm 2 \pi M_{D} \Omega^{2}\left(p \dot{y} / V_{r}\right)$,

$\ddot{q}+\varepsilon_{y} \Omega\left(q^{2}-1\right) \dot{q}+\Omega^{2} q=\Lambda_{y} \ddot{y}$,

in which

$M_{D}=C_{D 0} / 16 \pi^{2} \mathrm{St}^{2} \mu, M_{L}=C_{L 0} / 16 \pi^{2} \mathrm{St}^{2} \mu$,

$\mu=\left(m_{s}+m_{f}\right) / \rho D^{2}$,

$\lambda_{x}=2 \xi_{x} f^{*}+\gamma \Omega / \mu, \lambda_{y}=2 \xi_{y}+\gamma \Omega / \mu, f^{*}=\omega_{n x} / \omega_{n y}$,

$\Omega=\mathrm{St} V_{r}, m_{f}=\pi \rho D^{2} C_{M} / 4, m_{s}$ is the cylinder mass, $m_{f}$ the fluid added mass, $C_{M}$ the added mass coefficient assumed to be unity for a circular cylinder (Blevins, 1990), St the Strouhal number, $\gamma$ the stall parameter which is directly related to the sectional mean drag coefficient and assumed to be a constant equal to 0.8 (Facchinetti et al., 2004), and co-subscripts $x$ and $y$ identify properties in these directions. Note that the mass ratio definition in the literature is variable but the widely recognized one with $m^{*}=4 \mu / \pi-C_{M}$ is herein considered (Williamson and Govardhan, 2004).

In contrast to typical VIV models which consider a linear structural oscillator to describe the cylinder displacement (Gabbai and Benaroya, 2005), Eqs. (3) and (5) account for the effect of geometric nonlinearities (i.e. nonlinear stiffness or restoring force) of the oscillating cylinder. These equations are so-called Duffing-type oscillators (Nayfeh, 1993). Cubic nonlinear terms capture the effect of nonlinear stretching $\left(x^{3}, y^{3}\right)$ and physical cross-flow/in-line displacement coupling $\left(x y^{2}, x^{2} y\right)$, depending on the geometrical parameters $\left(\alpha_{x}, \alpha_{y}, \beta_{x}, \beta_{y}\right)$. Quadratic nonlinear terms have been found to be responsible for the figure-of-eight appearance associated with a 2:1 resonance condition (Srinil and Zanganeh, 2012). The coupling and interaction between the fluid and the structure is captured through all linear and nonlinear terms in the right-hand side of Eqs. (3)-(6). It is also worth remarking that, in Eqs. (3) and (5), the maximum cross-flow/in-line amplitudes are unaffected by the choice of $\theta$ since the associated velocities are trivial.

The analysis and prediction of coupled cross-flow/in-line VIV depend on a number of empirical coefficients $\left(\varepsilon_{x}, \varepsilon_{y}, \Lambda_{x}\right.$, $\left.\Lambda_{y}\right)$ and geometrical parameters $\left(\alpha_{x}, \alpha_{y}, \beta_{x}, \beta_{y}\right)$. Based on calibration with experimental results (Stappenbelt et al., 2007) with varying $m^{*}$ and $f^{*}=1$, it may be assumed that

$\varepsilon_{y}=0.00234 e^{\left(0.228 m_{y}^{*}\right)}$.

To reduce the time-consuming task involving the tuning of individual model coefficients, $\varepsilon_{x}=0.3, \Lambda_{x}=\Lambda_{y}=15$, and $\alpha_{x}=$ $\alpha_{y}=\beta_{x}=\beta_{y}=0.7$ are initially assumed in all $f^{*}$ cases, unless stated otherwise. Eqs. (3)-(6) are nonlinearly coupled and can be numerically solved by using a fourth-order Runge-Kutta scheme with an adaptive time step enabling solution convergence and stability, and with assigned initial conditions at $t=0$ of $x=y=0, p=q=2$ and zero velocities.

\section{EXTRACTION OF HYDRODYNAMIC COEFFICIENTS}

It has been observed by several experimental tests that, regardless of the specified $f^{*}$, most of the $x-y$ trajectories exhibit the figure-eight orbits (Dahl et al. 2006; 2010), depending on the corresponding in-line/cross-flow amplitudes, initial conditions, relative phases and the nearness of 2:1 resonant frequencies. These dual resonance characteristics result in the two-dimensional lock-in conditions. To extract the associated hydrodynamic properties for a 2-DOF VIV, a perfect dual resonance is herein assumed: both the cylinder cross-flow motion $y$ and the lift force $q$ are approximately sinusoidal at a common oscillating frequency $\omega$ whereas both the cylinder inline motion $x$ and the drag force $p$ are approximately sinusoidal at $2 \omega$. Accordingly, steady-state solutions of Eqs. (3)-(6) may be postulated as

$$
\begin{array}{ll}
x=x_{0} \sin \left(2 \omega t+\theta_{x}\right), & p=p_{0} \sin \left(2 \omega t+\theta_{p}\right), \\
y=y_{0} \sin \left(\omega t+\theta_{y}\right), & q=q_{0} \sin \left(\omega t+\theta_{q}\right),
\end{array}
$$

where $x_{0}, p_{0}, y_{0}$, and $q_{0}$ are dimensionless amplitudes, $\theta_{x}, \theta_{p}, \theta_{y}$, and $\theta_{q}$ are associated phases. By substituting Eqs. (9)-(10) into Eqs. (3)-(6), applying the harmonic balance method and neglecting higher-order nonlinear terms (although this may be invalid for a large amplitude motion), the analytical expressions for (i) the mean drag coefficient caused by VIV (due to the quadratic nonlinearities associated with the in-line cylinderwake interaction), (ii) the oscillating in-line force component in phase with the cylinder acceleration, (iii) the oscillating in-line force component in phase with the cylinder velocity, (iv) the oscillating transverse force component in phase with the cylinder acceleration and (v) the oscillating transverse force component in phase with the cylinder velocity, may be decomposed and derived, respectively, as follows

$$
\begin{aligned}
& C_{D V}=\mp \frac{8 \pi^{2} \mu}{V_{r}^{2}}\left(\pi \mathrm{St} M_{L} \Omega q_{0} y_{0} \omega \sin \left(\theta_{q}-\theta_{y}\right)\right), \\
& C_{a x}=\frac{8 \pi^{2} \mu}{V_{r}^{2}}\left[\begin{array}{l}
M_{D} \Omega^{2} p_{0} \cos \left(\theta_{p}-\theta_{x}\right) \mp \\
\pi \mathrm{St} M_{L} \Omega q_{0} y_{0} \omega \cos \left(\theta_{q}+\theta_{y}-\theta_{x}\right)
\end{array}\right], \\
& C_{v x}=\frac{8 \pi^{2} \mu}{V_{r}^{2}}\left[\begin{array}{l}
-M_{D} \Omega^{2} p_{0} \sin \left(\theta_{p}-\theta_{x}\right) \pm \\
\pi \mathrm{St} M_{L} \Omega q_{0} y_{0} \omega \sin \left(\theta_{q}+\theta_{y}-\theta_{x}\right)
\end{array}\right], \\
& C_{a y}=\frac{8 \pi^{2} \mu}{V_{r}^{2}}\left[\begin{array}{l}
M_{L} \Omega^{2} q_{0} \cos \left(\theta_{q}-\theta_{y}\right) \pm \\
\pi \mathrm{St} M_{D} \Omega p_{0} y_{0} \omega \cos \left(\theta_{p}-2 \theta_{y}\right)
\end{array}\right], \\
& C_{v y}=\frac{8 \pi^{2} \mu}{V_{r}^{2}}\left[\begin{array}{l}
-M_{L} \Omega^{2} q_{0} \sin \left(\theta_{q}-\theta_{y}\right) \mp \\
\pi \mathrm{St} M_{D} \Omega p_{0} y_{0} \omega \sin \left(\theta_{p}-2 \theta_{y}\right)
\end{array}\right] .
\end{aligned}
$$

These coefficients depend on the mass ratio, vibration amplitudes, relative phases, reduced velocity parameter and the common oscillating frequency. With varying $V_{r}(\Omega)$, the values of $p_{0}, q_{0}, x_{0}, y_{0}, \theta_{x}, \theta_{p}, \theta_{y}, \theta_{q}$, and $\omega$ are numerically obtained based on direct numerical integrations. 


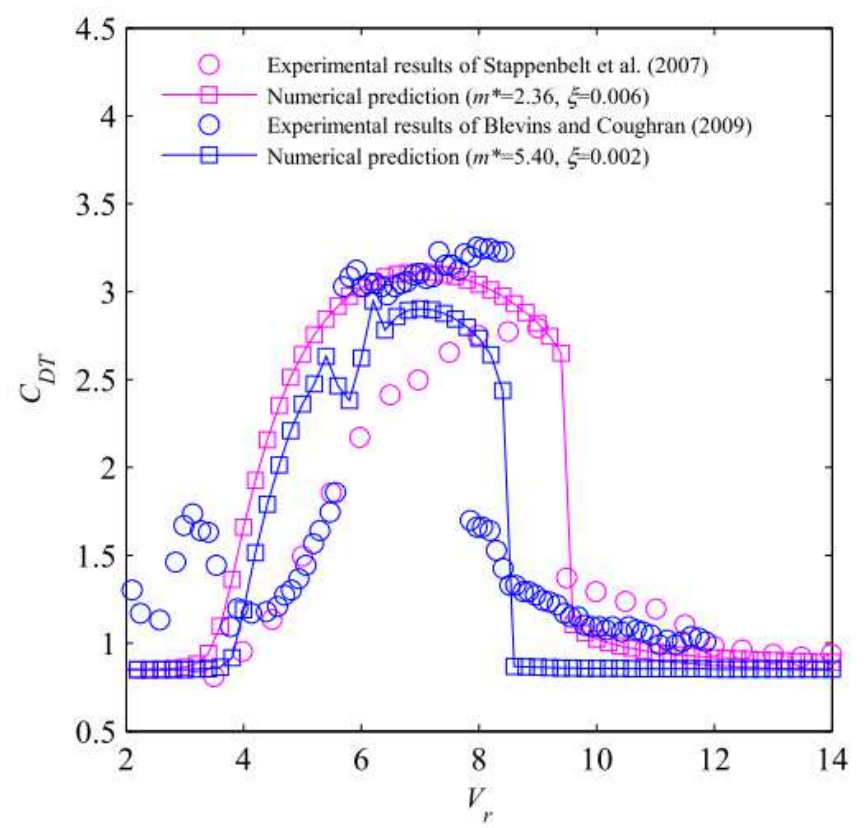

Figure 2 Comparison of numerical and experimental $C_{D T}$ with varying $V_{r}$ in the $f^{*}=1$ case

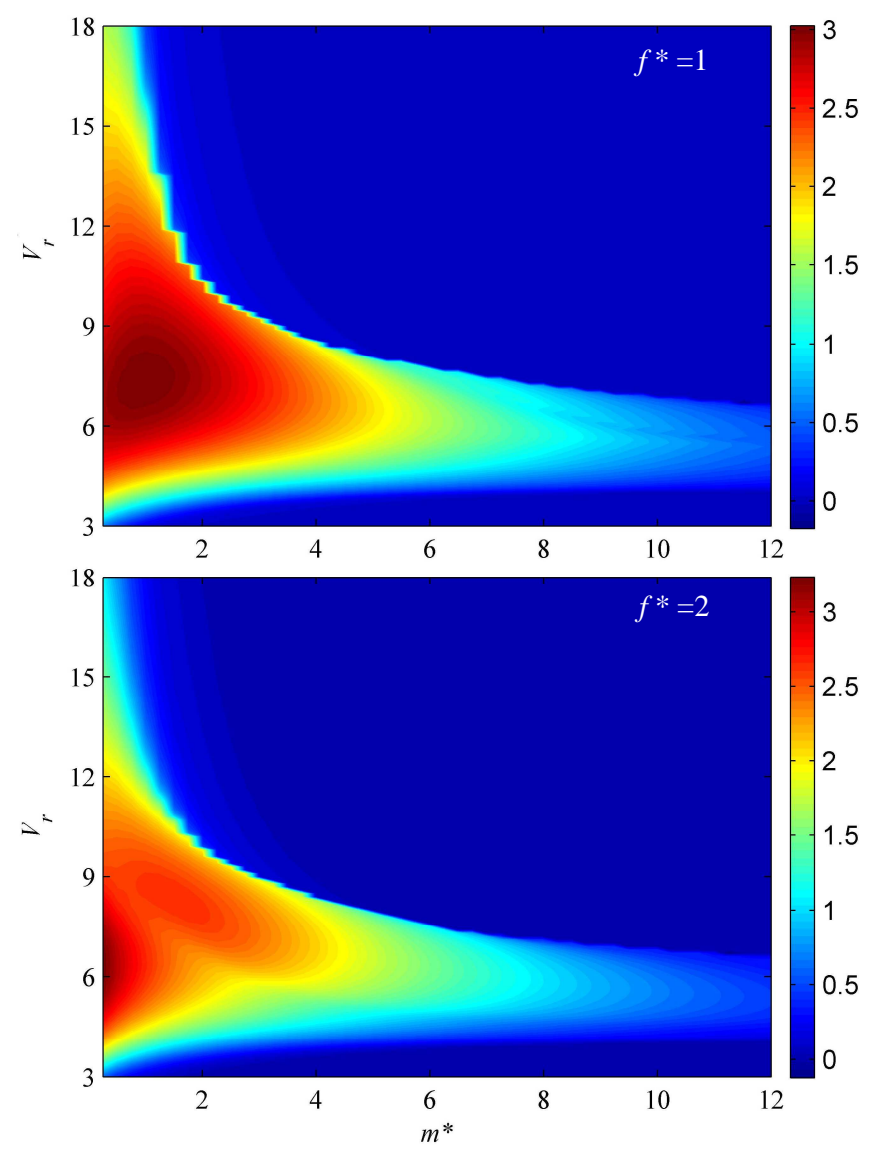

Figure 3 Variation of $C_{D T}$ with $m^{*}$ and $V_{r}$
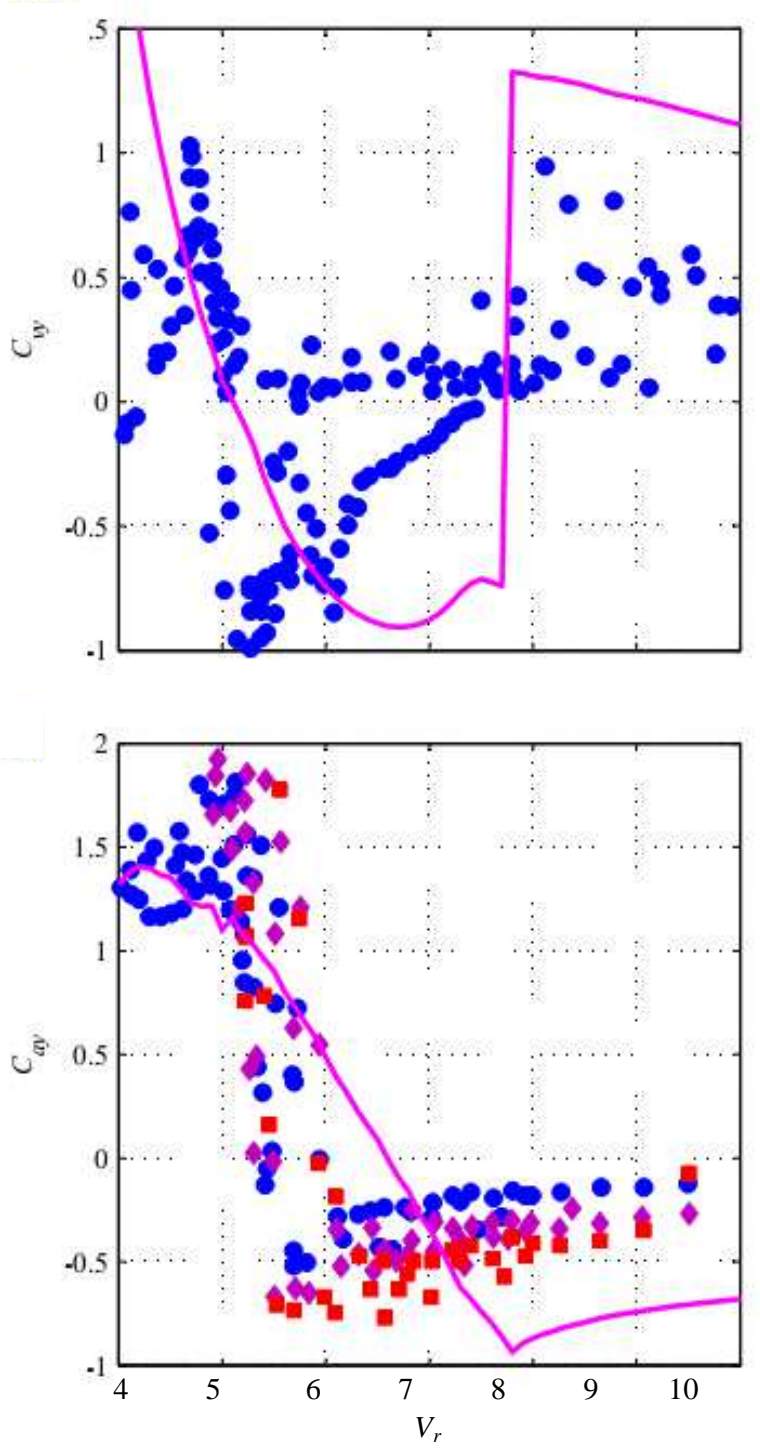

Figure 4 Variation of the oscillating lift force component in phase with the cylinder velocity $\left(C_{v y}\right)$ and acceleration $\left(C_{a y}\right)$ : lines denote numerical results, symbols denote experimental results compiled by Blevins (2009) and Sarpkaya (1995)

\section{RESULTS AND DISCUSSION}

Results of hydrodynamic coefficients extracted via the proposed mathematical model in the case of increasing $V_{r}$ and assumed clockwise $\theta$ (Fig. 1b and 1e) are now exemplified, by also focusing on the effect of $m^{*}$ and $f^{*}$. Figure 2 illustrates the variation of total mean drag coefficient $C_{D T}$ which comprises the mean drag coefficient of the associated stationary cylinder (taken as 0.83 as in Stappenbelt et al. 2007) and the mean drag coefficient due to VIV obtained from Eq. (11). Note that the phase difference is numerically observed such that $0<\theta_{q}-\theta_{y}<\pi$, leading to a positive sine term. Experimental models of Blevins and Coughran (2009) with $m^{*}=5.4$ and $\xi=0.002$ (cross-flow- 
only VIV), and Stappenbelt et al. (2007) with $m^{*}=2.36$ and $\xi=$ 0.006 (2-D VIV) are considered whose $C_{D T}$ results are also compared for a model validation purpose.

Overall, good qualitative and quantitative agreement of experimental and numerical results is found in Fig. 2 which suggests a maximum (average) $C_{D T}$ value of about 3.25 within a similar lock-in range $\left(4<V_{r}<10\right)$ where the response cylinder (cross-flow motion) and wake (lift force) amplitudes are maximized. Outside the lock-in regime, the stationary drag coefficient of 0.83 is the main component. Experimental results are more scattered. Similar to the amplitude response, a jump in the $C_{D T}$ plot is noticed experimentally as well as numerically: this suggests a possible hysteresis effect.

Figure 3 shows the effect of varying $m^{*}$ and $V_{r}$ on the total mean drag coefficient, for 2 cases of $f^{*}=1$ and $2(\xi=0.001)$. Results suggest a strong dependence of response amplitudes and lock-in ranges: the lower the $m^{*}$ value, the higher the $C_{D T}$ value. For a given $m^{*}$, larger $C_{D T}$ values are observed in the $f^{*}=1$ case, especially with $m^{*}<6$. This is due to the fact that crossflow amplitudes are slightly decreased as $f^{*}$ increases from 1 to 2 (Srinil and Zanganeh 2012). From numerical simulations, when $A_{y} / D$ is less than 1.1 , the value of $C_{D T}$ slightly changes with respect to increasing $V_{r}$. Thus, in this range, the mean drag function may be uniquely defined.

Figure 4 illustrates the variation of the oscillating transverse force component in phase with the cylinder velocity $\left(C_{v y}\right)$ and acceleration $\left(C_{a y}\right)$ with increasing $V_{r}$. Comparisons with a series of experimental forced vibration results (compiled by Sarpkaya 1995 and Blevins 2009) when specifying $m^{*}=6, \xi$ $=0.001$ and $f^{*}=1$ in numerical simulations is made. Both experimental and numerical results are in good agreement by showing that, within the initial and lower branches of the amplitude responses, $C_{v y}$ values are greater than zero, whereas in the upper branch associated with the lock-in range they are negative. The region of $C_{v y}<0$ is referred to as a negative damping region which indicates the excitation of the cylinder and how the energy is transferred from the fluid to the structure (Sarpkaya, 1995). When $C_{v y}$ achieves its minimum value (i.e. at the self-limiting state), the inertia component $C_{a y}$ tends to zero and changes sign across the resonance.

Overall, numerical results of minimum $C_{v y}$ and $C_{a y}$ occur at higher $V_{r}$ than experimental results according to their peak amplitudes. It should be noted, however, that experimental conditions were different as were their basic parameters such as the aspect ratios, Reynolds and Strouhal numbers.

With $f^{*}=1$ and $\xi=0.001$, Fig. 5 illustrates the effect of varying $m^{*}$ and $V_{r}$ on the oscillating transverse $\left(C_{v y}\right)$ and in-line $\left(C_{v x}\right)$ force components in phase with the cylinder velocity. It can be seen that both $C_{v y}$ and $C_{v x}$ plots exhibit similar diagrams and trends. For low $m^{*}<6(2), C_{v y}\left(C_{v x}\right)$ is negative within the lock-in region. As $m^{*}$ increases, both $C_{v y}$ and $C_{v x}$ increase and become no longer negative. Nevertheless, for the in-line response with $m^{*}$ being higher than 6 , there is no clear border between lock-in and non-synchronized region owing to the negligible in-line motion contribution to the 2-D VIV.
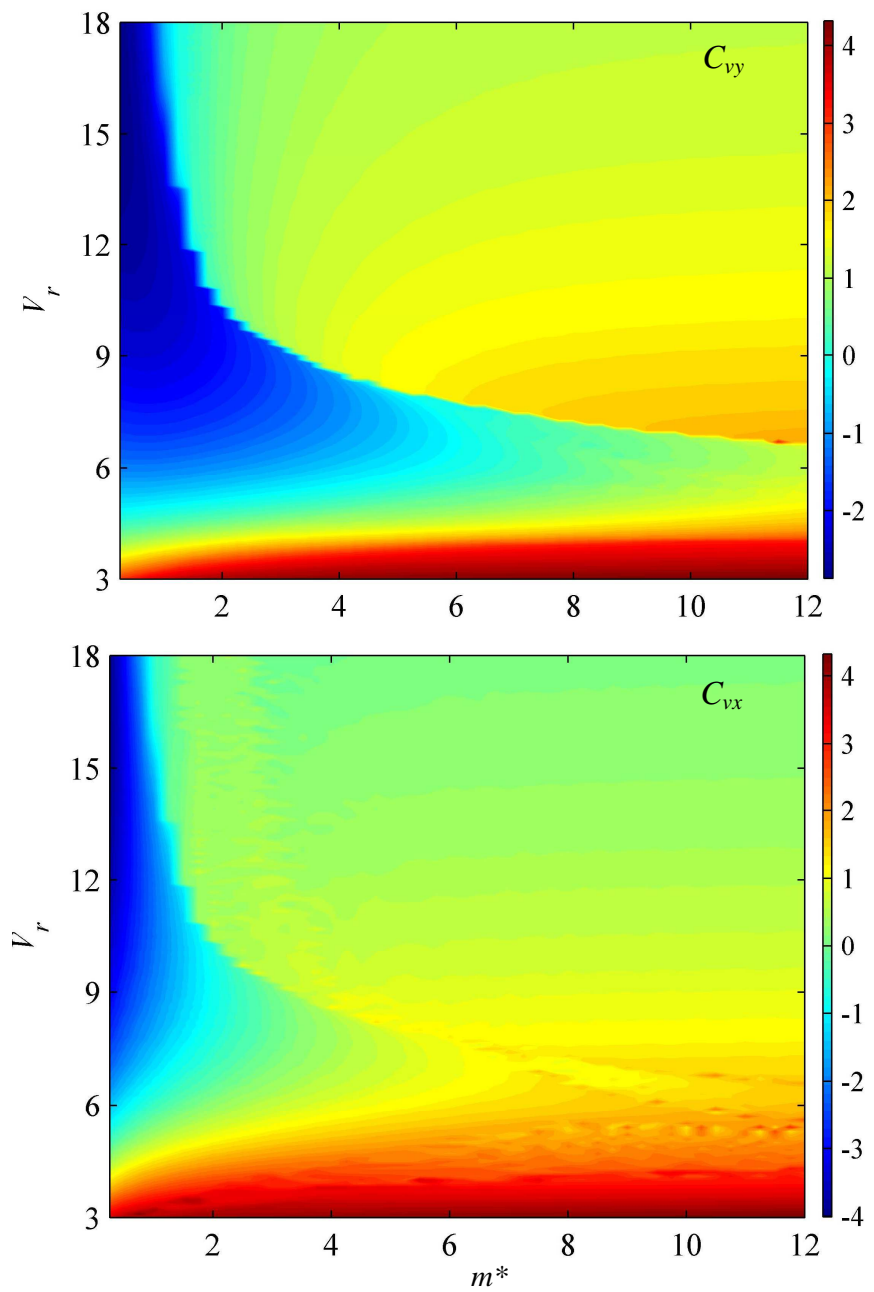

Figure 5 Variation of the oscillating force components in phase with the cylinder velocity with $f^{*}=1$

Moreover, Fig. 5 reveals that, as $m^{*}$ is less than a critical mass ratio, the absolute values of $C_{v y}$ and $C_{v x}$ increase as $V_{r}$ increases. As observed from recent experiments, such increasing range of the excitation coefficients at higher $V_{r}$ indicates the existence of the "lock-in forever" where associated response amplitudes persist (Govardhan and Wiiliamson, 2002).

The effect of varying $m^{*}$ and $V_{r}$ on values of $C_{a y}$ and $C_{a x}$ is now illustrated in Fig. 6 with two cases of $f^{*}=1$ and $2(\xi=$ $0.001)$. For $f^{*}=1, C_{a y}$ plots in a large range of $m^{*}$ show the typical transition from + to - values when increasing $V_{r}$. When increasing $m^{*}$, this transition range is very narrow, with both positive and negative values of $C_{a y}$ become decreased. These observations hold also in the case of $f^{*}=2$. On the contrary, there is a clear difference in the $C_{a x}$ plots between the cases of $f^{*}=1$ and 2 . In the former case, $C_{a x}$ values are generally negative, becoming nearly zero for large $m^{*}$. In the latter case, the $C_{a x}$ plots are similar to $C_{a y}$ plots where both negative and positive coefficients are realized, depending on $m^{*}$ and $V_{r}$. These results deserve further experimental validations. 

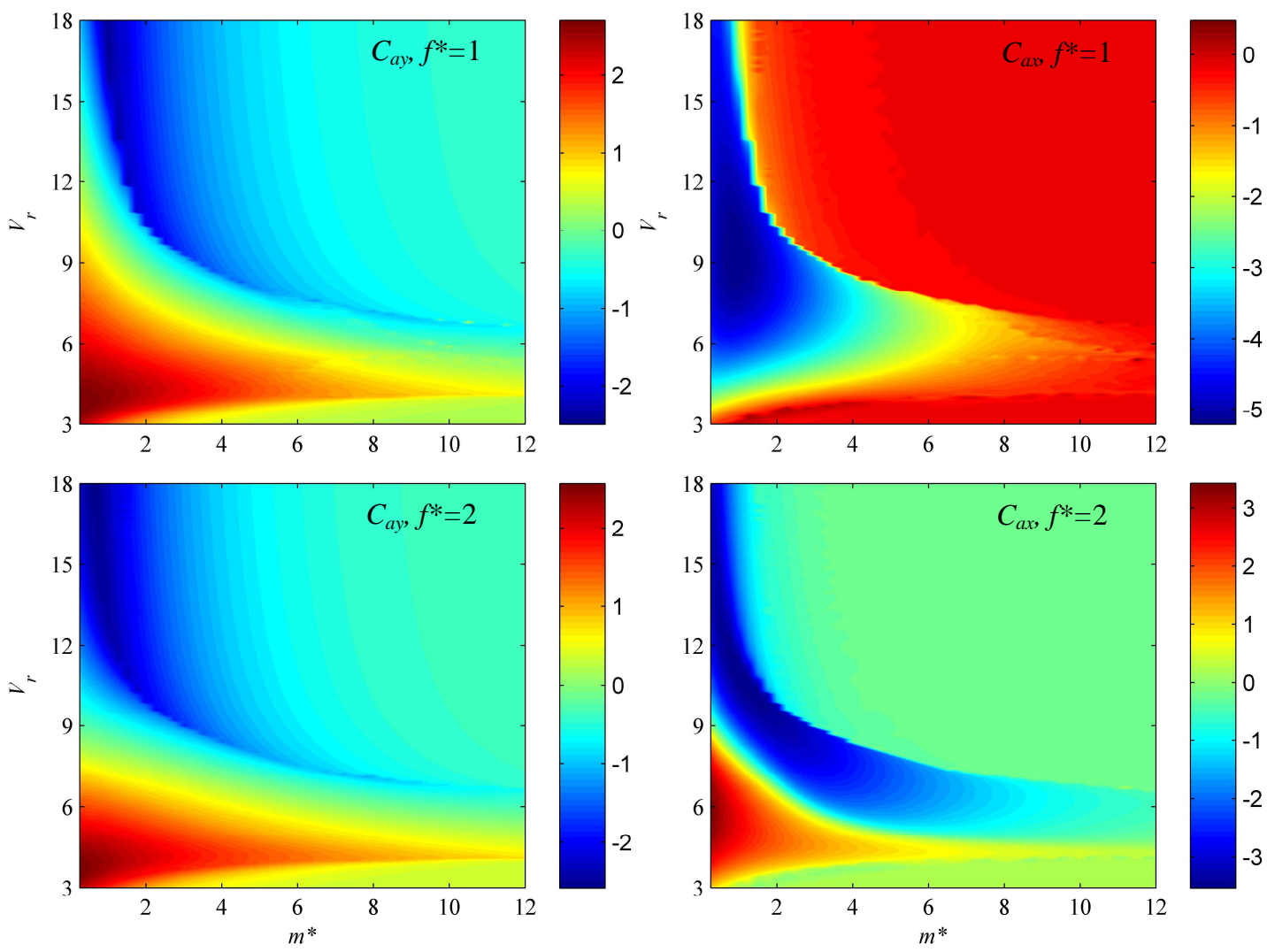

Figure 6 Variation of the oscillating lift and drag force component in phase with the cylinder acceleration

\section{CONCLUSIONS}

An advanced prediction model for a 2-DOF VIV involving cross-flow/in-line motions of an elastically-mounted circular cylinder in uniform flow has been proposed and investigated. The model is based on double Duffing-van der Pol (structurewake) oscillators, capturing the structural geometrical coupling and fluid-structure interaction effects through system cubic and quadratic nonlinearities. The combined analytical-numerical approach has been implemented to extract key hydrodynamic coefficients in both cross-flow and in-line directions. The hydrodynamic added mass, the mean drag and the oscillating transverse/in-line forces in phase with the velocity and acceleration of the cylinder are analyzed. Some comparisons with published experimental results have been made, proving a good agreement, although more experimental free-vibration investigations are needed. Numerical results highlight the effect of mass ratio, reduced flow velocity and cylinder in-line-tocross-flow natural frequency ratio on the values as well as the sign of hydrodynamic coefficients, in conjunction with the dependence on oscillation amplitudes (and relative phases) in both cross-flow and in-line directions. These model predictions could be correlated with the forced-vibration results and flow visualization studies, and applicable to the analysis of a flexible long cylinder involving a bi-directional multi-mode interaction.

\section{ACKNOWLEDGEMENTS}

The authors are grateful to the scholarships provided by the University of Strathclyde and the Scottish Funding Council through the Scottish Overseas Research Students Awards Scheme (SORSAS).

\section{REFERENCES}

Bearman, P.W., 2011. Circular cylinder wakes and vortexinduced vibrations. Journal of Fluids and Structures 27, 648658.

Blevins, R.D., 1990. Flow-Induced Vibrations. Van Nostrand Reinhold, New York.

Blevins, R.D., 2009. Models for vortex-induced vibration of cylinders based on measured forces. Journal of Fluids Engineering 131, 101203-9.

Blevins, R.D., Coughran, C.S., 2009. Experimental investigation of vortex-induced vibration in one and two dimensions with variable mass, damping, and Reynolds number. Journal of Fluids Engineering 131 (10), 101202-7.

Chaplin, J.R., Bearman, P.W., Cheng, Y., Fontaine, E., Graham, J.M.R., Herfjord, K., Huera Huarte, F.J., Isherwood, M., Lambrakos, K., Larsen, C.M., Meneghini, J.R., Moe, G., Pattenden, R.J., Triantafyllou, M.S., Willden, R.H.J., 2005. Blind predictions of laboratory measurements of vortex-induced 
vibrations of a tension riser. Journal of Fluids and Structures 21 (1), 25-40.

Currie, I.G., Turnbull, D.H., 1987. Streamwise oscillations of cylinders near the critical Reynolds number. Journal of Fluids and Structures 1 (2), 185-196.

Dahl, J.M., Hover, F.S., Triantafyllou, M.S., 2006. Twodegree-of-freedom vortex-induced vibrations using a force assisted apparatus. Journal of Fluids and Structures 22, 807818.

Dahl, J.M., Hover, F.S., Triantafyllou, M.S., Oakley, O.H., 2010. Dual resonance in vortex-induced vibrations at subcritical and supercritical Reynolds numbers, Journal of Fluid Mechanics, pp. 395-424.

Facchinetti, M.L., de Langre, E., Biolley, F., 2004. Coupling of structure and wake oscillators in vortex-induced vibrations. Journal of Fluids and Structures 19, 123-140.

Gabbai, R.D., Benaroya, H., 2005. An overview of modeling and experiments of vortex-induced vibration of circular cylinders. Journal of Sound and Vibration 282, 575616.

Govardhan, R., Williamson, C.H.K., 2002. Resonance forever: existence of a critical mass and an infinite regime of resonance in vortex-induced vibration. Journal of Fluid Mechanics 473, 147-166.

Jauvtis, N., Williamson, C.H.K., 2004. The effect of two degrees of freedom on vortex-induced vibration at low mass and damping. Journal of Fluid Mechanics 509, 23-62.

Nayfeh, A.H., 1993. Introduction to perturbation techniques. Wiley, New York.
Sarpkaya, T., 1995. Hydrodynamic damping, flow-induced oscillations, and biharmonic response. Journal of Offshore Mechanics and Arctic Engineering 117, 232-238.

Sarpkaya, T., 2004. A critical review of the intrinsic nature of vortex-induced vibrations. Journal of Fluids and Structures 19, 389-447.

Srinil, N., 2010. Multi-mode interactions in vortex-induced vibrations of flexible curved/straight structures with geometric nonlinearities. Journal of Fluids and Structures 26, 1098-1122.

Srinil, N., 2011. Analysis and prediction of vortex-induced vibrations of variable-tension vertical risers in linearly sheared currents. Applied Ocean Research 33, 41-53.

Srinil, N., Wiercigroch, M., O’Brien, P., 2009. Reducedorder modelling of vortex-induced vibration of catenary riser. Ocean Engineering 36, 1404-1414.

Srinil, N., Zanganeh, H., 2012. Modelling of coupled cross-flow/in-line vortex-induced vibrations using double Duffing and van der Pol oscillators. Ocean Engineering 53, 8397.

Stappenbelt, B., Lalji, F., Tan, G., 2007. Low mass ratio vortex-induced motion, The 16th Australasian Fluid Mechanics Conference, Gold Coast, Australia,, pp. 1491-1497.

Vandiver, J.K., Jong, J.Y., 1987. The relationship between in-line and cross-flow vortex-induced vibration of cylinders. Journal of Fluids and Structures 1, 381-399.

Wang, X.Q., So, R.M.C., Chan, K.T., 2003. A non-linear fluid force model for vortex-induced vibration of an elastic cylinder. Journal of Sound and Vibration 260, 287-305.

Williamson, C.H.K., Govardhan, R., 2004. Vortex-induced vibrations. Annual Review of Fluid Mechanics 36, 413-455. 